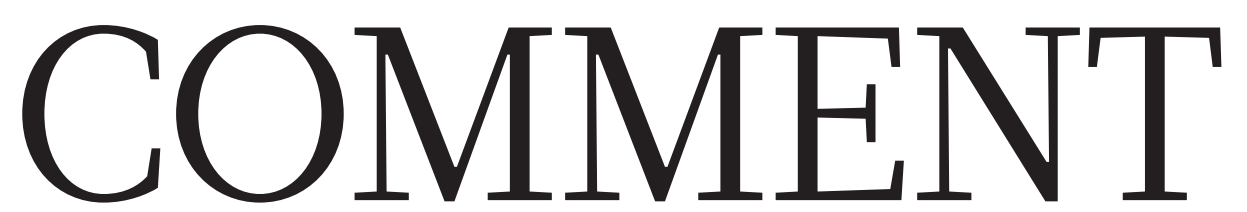

CLIMATE How are dust and soot affecting the warming of the planet? p.158
MACHINE LEARNING Artificial intelligence turns its untiring eye on art attribution $\mathbf{p . 1 6 1}$
MILITARY TECHNOLOGY US

surveillance project

shaped by 1998 movie $\mathbf{p} . \mathbf{6 2}$
ANTHROPOCENE Ernst Haeckel named humans' mark on the planet in $1868 \mathbf{p . 1 6 4}$

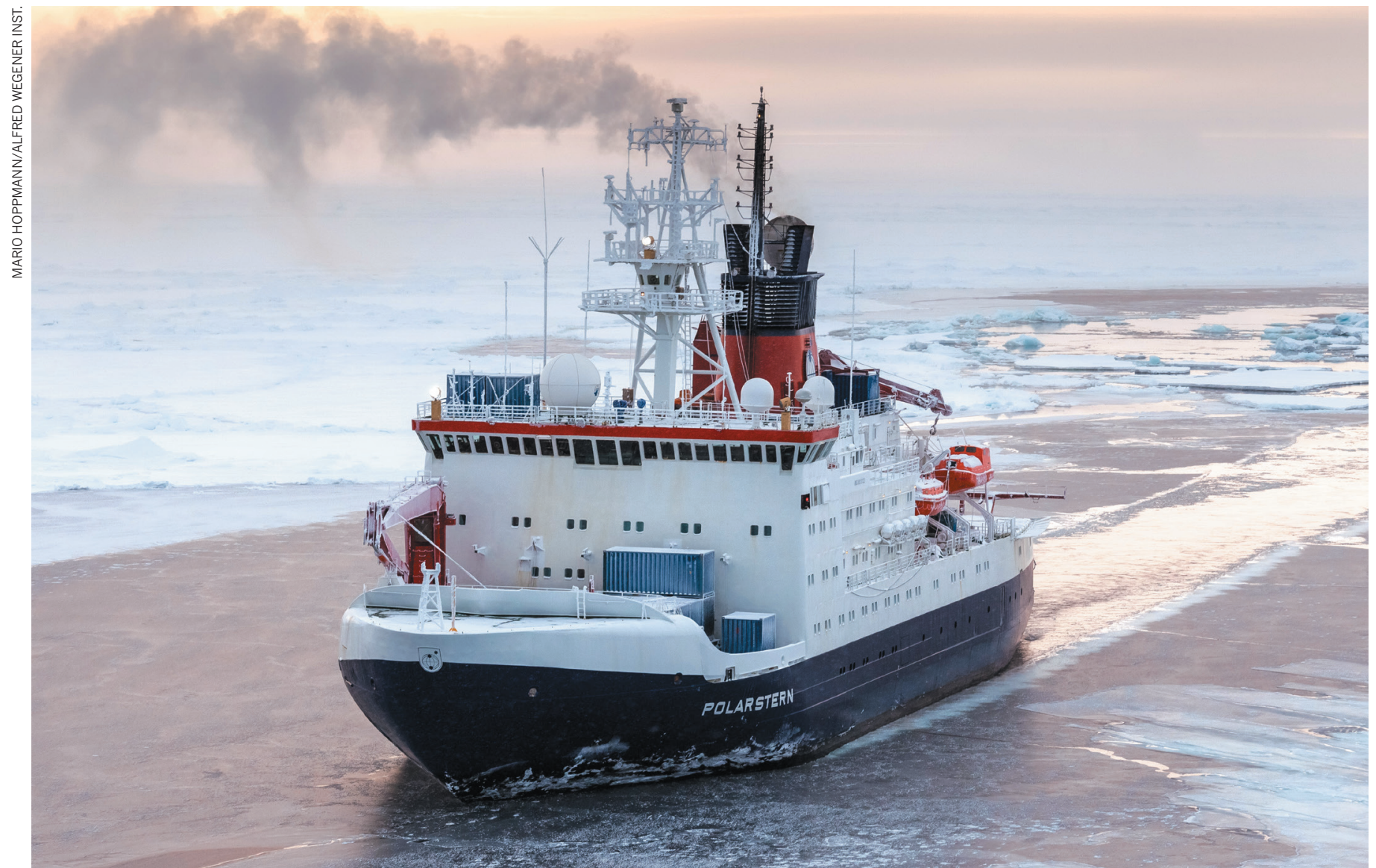

The German ship Polarstern was used in a research experiment that fertilized part of the Southern Ocean with iron in 2004.

\title{
Should we fertilize oceans or seed clouds? No one knows
}

Gather scientific evidence on the feasibility and risks of marine geoengineering to guide regulation of research, advise Philip Boyd and Chris Vivian.

$\mathrm{T}$ The climate clock is ticking. To turn it back, the world is putting its faith in 'negative-emissions technologies'. These would suck carbon dioxide out of the atmosphere and lock it up for centuries on the land, in the sea or beneath the sea floor (see 'Marine geoengineering'). Although such technologies are yet to be developed, they are nonetheless implicit in assessments by the Intergovernmental Panel on Climate Change (IPCC). To limit warming to $1.5^{\circ} \mathrm{C}$ compared to pre-industrial levels, as much as 20 billion tonnes (gigatonnes) of $\mathrm{CO}_{2}$ might need to be removed from the atmosphere each year until 2100 (ref. 1).

Storing carbon in the oceans sounds promising to some. The oceans are vast, and there could be fewer political trade-offs to deal with than on land. For example, fertilizing the water with iron would speed up the growth of phytoplankton and thus take up $\mathrm{CO}_{2}$, some of which would sink into the deep ocean as carbon when the organisms die. Another proposal is to spray seawater into the air to help form clouds that reflect sunlight and cool the planet.

Techniques such as these would need 


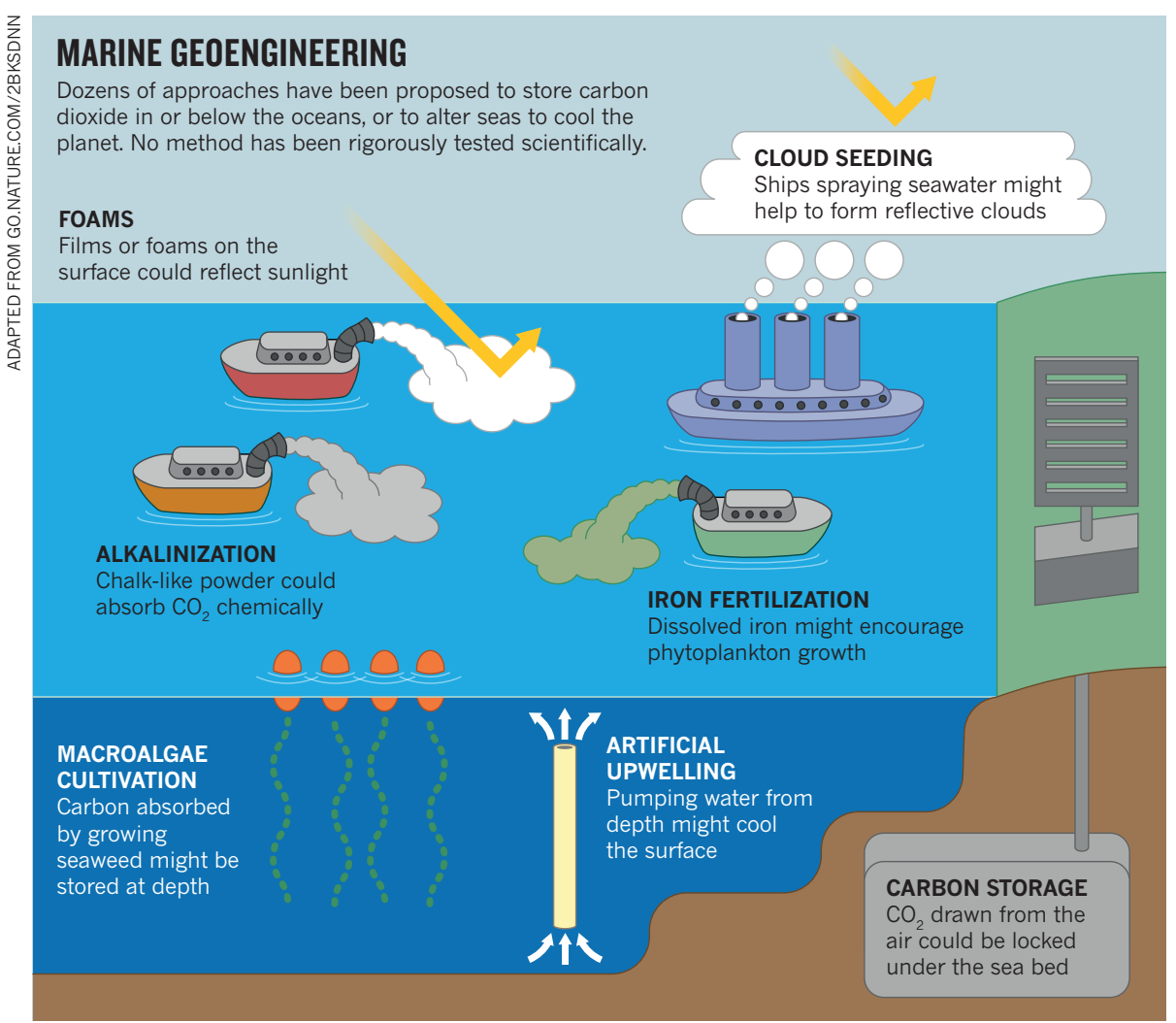

to be used on a massive scale to cap global warming at safe levels. For example, to mop up $\mathrm{CO}_{2}$ chemically, the entire Pacific Ocean would have to be sprinkled with one billion tonnes of powdered minerals similar to chalk. And these measures might be needed within a decade if emissions cuts fail and pressures mount on policymakers to act.

Little is known about the consequences. Scant research has been carried out for a range of reasons. The controversial nature of geoengineering divides researchers. And some research trials that have been funded have subsequently been abandoned, owing to a lack of rules for performing them and to conflicts of interest, such as patent applications (see Nature http://doi.org/hw2; 2012). Even basic tests of equipment haven't been done. Most of the preliminary studies have not been published in peer-reviewed academic journals.

This dearth of information is hampering the development of a global framework for regulating geoengineering research, despite more than a decade of debate. Researchers and policymakers need to know which negative-emissions technologies are worth investigating, and which will never work or are too damaging to pursue. The potential benefits and risks of the technologies need to be established before country leaders or companies decide to implement them prematurely.

As a first step, the United Nations Joint Group of Experts on the Scientific Aspects of Marine Environmental Protection (GESAMP) set up a working group in 2016 , which we co-chaired, to look at the potential

ecological and social impacts of various marine-geoengineering approaches. Its remit was to advise the International Maritime Organization (IMO) and parties to the 1996 Protocol to the Convention on the Prevention of Marine Pollution by Dumping of Wastes and Other Matter, 1972 (the London Protocol 1996). Our
"Techniques such as these would need to be used on a massive scale to cap global warming at safe levels." report was released in March 2019 (ref. 2). However, we struggled to identify techniques that are in need of regulation because the evidence trail was so poor. In the end, all we could do was to classify marine geoengineering research as either too insufficient or incomplete to inform a scientific assessment.

Here we call on advocates of geoengineering, from research to commerce, to build a body of basic scientific evidence within the next three years. This would enable policymakers to decide which methods to rule out and which hold potential. Geoengineering knowledge and regulation must advance in parallel.

\section{PATCHY PROGRESS}

The GESAMP working group examined 27 marine geoengineering ideas — from adding reflective foams to the surface of the ocean to burying carbon beneath the sea bed $^{2}$. Most of the research is in its embryonic stages. Some concepts, such as depositing crop wastes on the sea floor, have progressed little beyond thought experiments. A few have been studied in the lab or modelled on a computer. Fewer than ten pilot studies have been done in the field.

Glaring research gaps abound. For example, the idea of using a fine spray of seawater or other aerosols to thicken or seed clouds above the ocean - similar to the trails created by emissions from ships - has featured widely in the media. No one has reported on any attempt to spray fine droplets of natural seawater (although there have been lab experiments on manufactured salt water ${ }^{3}$ ). But seawater is full of tiny organisms and organic material that could clog a sprayer (as pointed out in the GESAMP report ${ }^{2}$ by working-group member John Cullen, an oceanographer at Dalhousie University in Halifax, Canada).

A lack of funding is not the main reason for the research gaps. Although there have been few funding programmes targeted at marine-geoengineering experiments and modelling so far, many basic tests are cheap and can be done in the lab - for instance, assessing whether impurities in mineral powders are toxic to marine life. And a range of negative-emissions technologies, such as enhanced weathering of rocks to increase ocean alkalinity, are already being funded in targeted research programmes, including one in the United Kingdom. Other streams of research, such as modelling, are under way in Germany, and a call for research proposals has been made in Japan. Private money is being invested in some marine approaches, such as a proposed pilot study of the impacts of iron fertilization on fisheries off Chile. However, that project has stalled, largely because of a lack of support from scientists (see Nature 545, 393-394; 2017).

Another problem is that many geoengineering proposals and analyses are found on transient websites, not in peer-reviewed journals. For example, only half of the web links to ideas, plans and documents cited in a detailed 2009 synthesis study of marine-geoengineering approaches ${ }^{4}$ still worked when we examined them in 2018. By contrast, academic and intergovernmental documents from that era are easy to find.

Again, the reasons for this are unclear, but could include inadequate funding, privacy concerns about disclosing details of the methods, and maintenance of proprietary rights over technologies. Some scientists worry that even starting geoengineering research or reporting results could lead to deployment of inadequately studied approaches ${ }^{5}$.

Yet it is essential that investigations are solidly researched, openly discussed and made readily available, as demonstrated by the most-studied geoengineering approach, ocean iron fertilization. Much of the work drew from ocean biogeochemistry and has 
involved lab experiments, pilot studies in the Southern Ocean and modelling across ocean basins. All of this activity showed that the method will not work as anticipated ${ }^{6}$. Fertilizing 1,000 square kilometres of the upper ocean would increase the growth of phytoplankton but could have alarming side effects. For example, sinking algae could release methane, a greenhouse gas that is many times more potent than $\mathrm{CO}_{2}$.

This body of research convinced policymakers to intervene. In 2008, parties to the UN Convention on Biological Diversity agreed voluntarily to stop large-scale iron fertilization experiments from going ahead in the oceans without scientific risk assessments, controls and regulation (see Nature 453, 704; 2008). In 2013, the London Protocol added legally binding amendments to regulate ocean iron fertilization, but they have yet to enter into force.

No other marine-geoengineering methods have got far enough to prompt similar regulation. Yet techniques such as ocean alkalinization are likely to have large-scale impacts that are similar to those of iron fertilization ${ }^{2}$. Models highlight potential problems with other methods: simulations have revealed, for example, that pumping nutrient-rich cold waters to the surface of the Pacific could lower the temperature of overlying air and help to cool the planet, but at the expense of creating a vast low-oxygen zone that would threaten fisheries ${ }^{7}$.

\section{THE WAY FORWARD}

As climate dangers mount, marine geoengineering needs a body of evidence to guide research and regulation. We suggest that the UN Framework Convention on Climate Change or the International Science Council should take the lead by promoting four steps.

First, to build the foundations, researchers must identify and start to fill key knowledge gaps ${ }^{2}$. Many basic questions can be answered with minimal funding in lab settings and without new legislation. For example, will mineral powders make seawater less transparent or will they enter the food web, as tiny specks of plastic have done? By how much will reflective foams on the surface reduce photosynthesis? How long will the foams last and how will the wind affect their spread?

A shared bank of standard models of the oceans, similar to those used by the IPCC to ensure consensus across climate models, should be developed through initiatives such as the Carbon Dioxide Removal Model Intercomparison Project (see go.nature. com/2wevzhj). Links should be tightened between ocean modellers and related research communities such as SOLAS (Surface Ocean Lower Atmosphere Study), which in April held a workshop on this topic in Sapporo, Japan.

All results must be published in archived journals and repositories. Standards must be



A phytoplankton bloom in the North Sea off the east coast of Scotland, UK, in 2008.

developed for reporting details of methods, so that studies can be repeated or compared under a range of conditions.

Second, the potential benefits and drawbacks of each geoengineering method need to be assessed and ranked. Such a portfolio, updated as evidence accrues, should be held by an international organization such as GESAMP. Benefits should include evidence of efficient and permanent removal of $\mathrm{CO}_{2}$ with minimal side effects. Drawbacks might include unanticipated difficulties in scaling up the technology and unintended adverse consequences, such as the enhancement of populations of some toxic phytoplankton species by ocean iron fertilization ${ }^{8}$.

Third, researchers and policymakers should develop scientific criteria for evaluating risks, and devise a set of tests that experiments must pass before they are permitted. These should encompass the intended and unintended consequences of the work, as well as the propagation of risk as more ambitious research is done - for example, when a pilot study in a lab flask moves gradually to other enclosed environments (such as a large-volume incubator) and then to the open sea. Policymakers will need to decide which methods merit further consideration, and which should be dismissed as impractical or as unacceptably risky.

Fourth, if such tests are passed, research and regulation should proceed in parallel. The London Protocol is a good starting point for governing interventions in the oceans. Researchers and policymakers will need to devise an adaptive framework for gathering, evaluating and responding to evidence for all candidate geoengineering approaches, including marine methods. Governance of research must be informed by a wide range of outcomes.
Resources can then be targeted at the most promising areas.

Adaptive forms of governance that centre on responsible research and innovation ${ }^{9}$ have been applied to other emerging technologies, such as synthetic biology and nanotechnol$\operatorname{ogy}^{10}$. A similar approach for geoengineering will enable scientists to put forward a scientifically sound subset of approaches that can be scrutinized through legal, socio-economic and geopolitical lenses over the next few years.

Philip Boyd is professor of marine biogeochemistry at the Institute for Marine and Antarctic Studies, University of Tasmania, Hobart, Australia. Chris Vivian is retired and formerly worked as a national marine adviser at the Centre for Environment, Fisheries and Aquaculture Science, Lowestoft, UK. e-mail:philip.boyd@utas.edu.au

1. Intergovernmental Panel on Climate Change. Summary for Policymakers. In Global Warming of $1.5^{\circ} \mathrm{C}$ (IPCC, 2018)

2. GESAMP Working Group 41. High Level Review of a Wide Range of Proposed Marine Geoengineering Techniques (eds Boyd, P. W. \& Vivian, C. M. G.) GESAMP Rep. Stud. No. 98 (International Maritime Organization, 2019)

3. Cooper, G. et al. Phil. Trans. R. Soc. A 372, 20140055 (2014)

4. Strong, A. L., Cullen, J. J. \& Chisholm, S. W. Oceanography 22, 236-261 (2009).

5. Schäfer, S. et al. The European Transdisciplinary Assessment of Climate Engineering (EUTRACE): Removing Greenhouse Gases from the Atmosphere and Reflecting Sunlight away from Earth (Institute for Advanced Sustainability Studies Potsdam, 2015).

6. Boyd, P. W. et al. Science 315, 612-617 (2007).

7. Keller, D. P., Feng, E. Y. \& Oschlies, A. Nature Commun. 5, 3304 (2014).

8. Silver, M. W. et al. Proc. Natl Acad. Sci. USA 107 20762-20767 (2010).

9. Genus, A. \& Stirling, A. Res. Policy 47, 61-69 (2018).

10.Kerr, A., Hill, R. L. \& Till, C. Technol. Soc. 52, 24-31 (2018). 